\title{
On the formation of black silicon in SF6-02 plasma: The clear, oxidize, remove, and etch (CORE) sequence and black silicon on demand
}

\author{
Nguyen, Vy Thi Hoang; Jensen, Flemming; Hübner, Jörg; Leussink, Pele; Jansen, Henri
}

Published in:

Journal of Vacuum Science \& Technology. A. Vacuum, Surfaces, and Films

Link to article, DOI:

$10.1116 / 6.0000196$

Publication date:

2020

Document Version

Publisher's PDF, also known as Version of record

Link back to DTU Orbit

Citation (APA):

Nguyen, V. T. H., Jensen, F., Hübner, J., Leussink, P., \& Jansen, H. (2020). On the formation of black silicon in $\mathrm{SF}^{-} \mathrm{O}_{2}$ plasma: The clear, oxidize, remove, and etch (CORE) sequence and black silicon on demand. Journal of Vachum Science \& Technology. A. Vacuum, Surfaces, and Films, 38(4), [043004]. https://doi.org/10.1116/6.0000196

\section{General rights}

Copyright and moral rights for the publications made accessible in the public portal are retained by the authors and/or other copyright owners and it is a condition of accessing publications that users recognise and abide by the legal requirements associated with these rights.

- Users may download and print one copy of any publication from the public portal for the purpose of private study or research.

- You may not further distribute the material or use it for any profit-making activity or commercial gain

- You may freely distribute the URL identifying the publication in the public portal 


\section{On the formation of black silicon in $\mathrm{SF}_{6^{-}}$ $\mathrm{O}_{2}$ plasma: The clear, oxidize, remove, and etch (CORE) sequence and black silicon on demand}

Cite as: J. Vac. Sci. Technol. A 38, 043004 (2020); https://doi.org/10.1116/6.0000196

Submitted: 16 March 2020 . Accepted: 27 May 2020 . Published Online: 12 June 2020

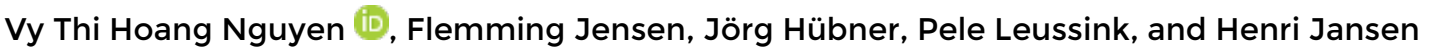



\title{
On the formation of black silicon in $\mathrm{SF}_{6}-\mathrm{O}_{2}$ plasma: The clear, oxidize, remove, and etch (CORE) sequence and black silicon on demand
}

Cite as: J. Vac. Sci. Technol. A 38, 043004 (2020); doi: $10.1116 / 6.0000196$

Submitted: 16 March 2020 . Accepted: 27 May 2020 .

Published Online: 12 June 2020

Vy Thi Hoang Nguyen, ${ }^{1}$ (D) Flemming Jensen, ${ }^{7}$ Jörg Hübner, ${ }^{7}$ Pele Leussink, ${ }^{2}$ and Henri Jansen ${ }^{7, a)}$

\begin{abstract}
AFFILIATIONS
${ }^{7}$ DTU Nanolab, Technical University of Denmark, Ørsteds Plads, Building 347, 2800 Kgs. Lyngby, Denmark

${ }^{2}$ MESA+ Nanolab, University of Twente, Drienerlolaan 5, 7522 NB Enschede, Netherlands
\end{abstract}



\begin{abstract}
Black silicon (BSi or silicon micro/nanograss) is a frequently encountered phenomenon in highly directional etching of silicon using mainstream plasma etch tools. The appearance of BSi in most studies is considered to be caused by micromasks unintentionally present on the silicon surface that locally prevent silicon from etching. Particularly, under highly directional and selective plasma etch conditions, these chaotically arranged micromasks develop into tall grasslike structures that will absorb incoming light and make the etched silicon appear black. There are many different sources that might contribute to the formation of BSi. Most of them can be prevented by proper pretreatment of the surface and careful control of the etch parameters. However, the masking related to the in situ plasma passivation (typically FC- or O-species) and insufficient ion etching of this layer causing residues at horizontal surfaces remains a resilient issue that is difficult to control or predict. This study is built on a recently developed highly directional etch procedure called CORE (meaning Clear, Oxidize, Remove, and Etch) in which the usual FC inhibitor of the Bosch process is replaced by oxygen. Due to the self-limiting property of the oxidation step, the formation and controllability of BSi in the CORE sequence is different from how BSi presents itself in the FC-based sequences. In this work, the effects of different process parameters on the creation of masks and formation of BSi are carefully investigated. The authors show that the time in the removal (R) step of the passivating oxide layer in tight combination with the undercut time in the isotropic etch (E) step are the most important parameters to consider. By manipulating these two parameters and utilizing the self-limiting property of the oxidation (O) step, the CORE process can easily be modified to create either BSi-full or BSi-free surfaces independent of the aspect ratio of the etching features. The latter distinguishes the BSi formation clearly from other directional processes. The proposed CORE process thus provides the authors a versatile tool for creating BSi anywhere anytime or-as we call it-"BSi on Demand."
\end{abstract}

Published under license by AVS. https://doi.org/10.1116/6.0000196

\section{INTRODUCTION}

The topography of silicon surfaces controls many of its physical, chemical, and biological properties. Particularly, texturing at the nanoscale has shown to effectively reduce the reflection of the visible light due to enhanced absorption and scattering. Because of the almost perfect light absorption, this type of silicon has been given the appropriate name of black silicon (BSi) and has found a prominent place in photovoltaic applications. ${ }^{1-4}$ The strongly increased surface area has also shown attractive opportunities in chemical and optical devices. $^{5-7}$ In addition, another already established characteristic of
$\mathrm{BSi}$ is its ability to prevent cell growth and as such creates antibacterial, antifogging, and self-cleaning surfaces. ${ }^{8-10}$

Even though the appearance of BSi possesses many beneficial features, the formation is considered to be a downside in most MEMS-related applications. ${ }^{11-20}$ It is consequently of importance to control the onset and subsequent evolution of BSi to either enhance or reduce its appearance. Therefore, it is helpful to find the root cause of BSi formation, and many studies have been devoted to find this. However, the onset of BSi is still highly debated, and many studies have documented and explained the problem differently. They can be grouped into three main categories: 
Fluorocarbon-related sources: (1) FC deposits from the reactor walls that lose contact and drop particles on the silicon surface ${ }^{21,22}$ and (2) plasma deposition of the requested FC inhibitor on the etching features, but insufficient removal from the horizontal surfaces by ion impact (puncture convolution) leaving residues. ${ }^{23}$

Oxygen-related sources: (3) oxide participants/clusters/defects/ impurities inside, e.g., Czochralski $\mathrm{Si}$, (4) nonvolatile $\mathrm{SiO}_{\mathrm{x}} \mathrm{F}_{\mathrm{y}}$ particles arriving from the plasma as a result of oxidizing $\mathrm{SiF}_{4}$ reaction products (plasma dust), ${ }^{20,24-27}$ (5) redeposit from sputtered $\mathrm{SiO}_{\mathrm{x}}$ that has formed at horizontal Si surfaces, (6) inhomogeneous removal of the initial native oxide, ${ }^{20,24,28}$ and (7) plasma oxidation of the Si surface and insufficient removal by ion impact leaving residues. $^{24,29}$

Other sources: (8) "Dirty" wafers due to previous process steps (e.g., resist residues or scum), ${ }^{24}$ (9) mask sputtering and subsequent redepositing, ${ }^{24}(10)$ nonvolatile sulfur compounds $\left(\mathrm{SO}_{\mathrm{x}} \mathrm{F}_{\mathrm{y}}\right)$ formed in the plasma bulk, (11) $\mathrm{AlF}_{3}$ particles sputtered from the reactor walls or from the wafer clamp, ${ }^{21,30}$ (12) natural roughness of the silicon surface causing self-shadowing, ${ }^{31}$ and (13) temperaturedependent amplification of particle diffusion along the surface. ${ }^{32}$

So, it is commonly believed that the formation of $\mathrm{BSi}$ is mainly caused by particles unintentionally deposited on a silicon surface or by remaining residues due to an incomplete removal of a passivation layer. Once present on the surface these particles or residues act like tiny masks and locally prevent silicon from further etching. The evolution of these micromasks into long pointy nanostructures is more pronounced when operating with an anisotropic etch recipe. Most of the self-inflicted masks listed above can be easily prevented by proper pretreatments and carefully controlling the etch conditions, except for those related to the insufficient bottom removal of the plasma passivation using fluorocarbon (\#2) or oxygen (\#7) that leaves residues. More specifically, the formation of grass in the FC related processes (\#2) is often more pronounced in large open areas than in high aspect ratio structures and is highly time-dependent. ${ }^{23}$ The main reason is the larger influx of passivating species in the open areas as will be explained further on. In this study, we replace the conventional Bosch process by a recently developed plasma etch process named CORE (Clear, Oxidize, Remove, and Etch) in which the $\mathrm{C}_{4} \mathrm{~F}_{8}$ passivation cycle is replaced by an $\mathrm{O}_{2}$ cycle. $^{33}$ The oxide thickness at the trench bottom is less dependent on the aspect ratio because the plasma oxidation is experimentally found to be self-limiting in our previous paper. ${ }^{33}$ In short, during plasma oxidation, the growing oxide film will slow down the oxidation process logarithmically and effectively limit its thickness to about $3 \mathrm{~nm}$ or less. Consequently, all the horizontal surfaces will be cleared from the passivation in approximately the same R-time. Using CORE, we demonstrate the possibility to control the resilient BSi initiator while keeping the ability to faithfully etch the intended pattern anisotropically. This finding has provided the authors a tool to manipulate and control BSi formation at will and independent of the aspect ratio of the etching features or time; hence, it is called "Black Silicon on Demand."

\section{MATERIALS AND METHODS}

Silicon wafers (150 mm Czochralski, n-doped, $\langle 100\rangle$ orientation) are coated with $1.5 \mu \mathrm{m}$ thick positive photoresist (AZ MiR
701, Merck) using a spin coating system (Gamma 2M, Süss MicroTech). Subsequently, patterns are defined by maskless UV-lithography (MLA150, Heidelberg Instruments), which is a direct writing system with a high power laser light source. The exposure dose of $180 \mathrm{~mJ} \mathrm{~cm}^{-2}$ will result in a resolution of around $600 \mathrm{~nm}$. Then, the wafers are developed in AZ726 MIF (AZ Electronic Materials), rinsed in de-ionized water, and dried by spin coating with a gentle nitrogen stream. To verify the critical dimension of the exposed patterns, the samples are inspected using an optical microscope (Nikon eclipse L200) that allows a measurement resolution of $200 \mathrm{~nm}$. Then, most wafers receive a short descum to remove any surface resist residue (preventing \#8). For this, a barrel etcher including a Faraday cage (Tepla 300 Semi-Auto) is used for $10 \mathrm{~min}$ at room temperature with $500 \mathrm{SCCM} \mathrm{O} \mathrm{O}_{2}$ at $1 \mathrm{mbar}$ and $150 \mathrm{~W}$. Subsequently, the samples are cleaved manually into pieces of around $1 \times 1 \mathrm{~cm}^{2}$ and attached on a $150 \mathrm{~mm}$ silicon carrier wafer by a small drop of Galden PFPE fluid (Solvay Solexis SpA). The PFPE fluid is a chemically inert perfluoropolyether vacuum oil with good thermal conductivity. Finally, the etch process is performed in a commercial available plasma etch system (Pegasus, SPTS). After etching, the samples are characterized using a scanning electron microscope (Supra V60, Zeiss) that allows for a $10 \mathrm{~nm}$ resolution.

The procedure to create directional profiles with $\mathrm{BSi}$ on demand is a recently developed four-step CORE sequence as schematically shown in Fig. 1. CORE uses a sequence of sulfurhexafluoride $\left(\mathrm{SF}_{6}\right)$ plasma to etch silicon (the E-step), repeatedly alternated with oxygen $\left(\mathrm{O}_{2}\right)$ plasma to passivate the sidewall of the etching features (the $\mathrm{O}$-step). Both steps have a very low bias in order to minimize the mask erosion. The R-step is included after the O-step to remove the passivation layer from the horizontal surfaces completely but leaving the vertical wall passivation intact. This step operates at a very low pressure to create a high plasma potential that will combine with the DC self-bias to enhance the ion energy for the directional removal. The C-step is less evident, but it is added as a purge step to remove the $\mathrm{SF}_{6}$ species from the reactor before the $\mathrm{O}$-radicals from the next $\mathrm{O}$-step arrive. Finally, the plasma is solely generated by the platen source (i.e., capacitively coupled plasma) as the coil source (i.e., inductively coupled plasma) without a protecting Faraday cage has a substantial risk of generating $\mathrm{AlF}_{\mathrm{x}}$ particles (thus preventing \#11) especially at low pressure.

\section{RESULTS AND DISCUSSION}

\section{A. Formation of BSi in the CORE process}

The CORE sequence has been shown to enable the etching of nanostructures having straight, smooth sidewalls with low (resist) mask erosion and undercutting. ${ }^{33}$ Unlike the Bosch process, ${ }^{34-37}$ the CORE sequence has no pileup of inhibitor deposits at the top of etching features. This is because the passivation layer originates from the silicon oxidation with a limited thickness as the growing silicon dioxide acts as a diffusion barrier. This feature makes the procedure outstanding for high aspect ratio or nanoscale directional etching. In addition, the bottom appears either everywhere clean (i.e., smooth inside high aspect ratio features and in the large open field area) or everywhere roughened. To study this atypical BSi formation behavior, this paper focuses on the onset of BSi formation in high aspect features and open field areas. The main difference 


\begin{tabular}{|l|c|c|c|c|}
\hline \multicolumn{3}{c|}{ CORE } & \multicolumn{3}{c|}{ Clear } & Oxidise & Remove & Etch \\
\hline Time (s) & 4 & 3 & 20 & 73 \\
\hline Pressure & & & & \\
\hline $\mathrm{O}_{2}$ & & & & \\
\hline Power & & & & \\
\hline Self-Bias & & & & \\
\hline SF $_{6}$ & & & & \\
\hline
\end{tabular}

\begin{tabular}{|l|c|c|c|c|}
\hline CORE & C & O & R & E \\
\hline Time (s) & 4 & 3 & 20 & 73 \\
\hline Pressure (mT) & 0.8 & 50 & 0.2 & 50 \\
\hline $\mathrm{O}_{2}(\mathrm{sccm})$ & 50 & 50 & 0 & 0 \\
\hline Platen Power (W) & 0 & 10 & 10 & 10 \\
\hline DC Self-Bias (V) & 0 & 0 & 4 & 0 \\
\hline SF $_{6}(\mathrm{sccm})$ & 0 & 0 & 5 & 15 \\
\hline
\end{tabular}

FIG. 1. Typical fine-tuned CORE cycle for nanoscale etching.

between the FC- and O-related sequences on how the deposition mechanism affects the overall BSi formation will be explained next.

As illustrated in Fig. 2, the directional sequence can be separated into three basic steps: passivation with FC- or O-species (left), removal of this layer using an ionic flux (middle), and silicon etching using F-radicals (right). All three steps have their own aspect ratio dependent characteristics. For example, during the Bosch deposition step [Fig. 2(a1)], the arrival cone of the FC
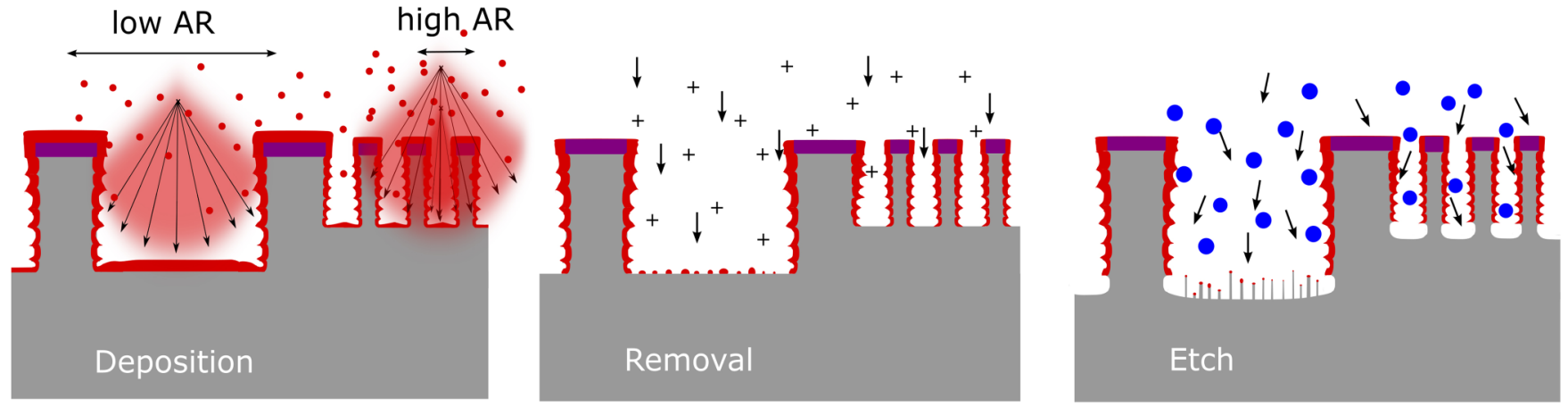

\section{a. Bosch process}
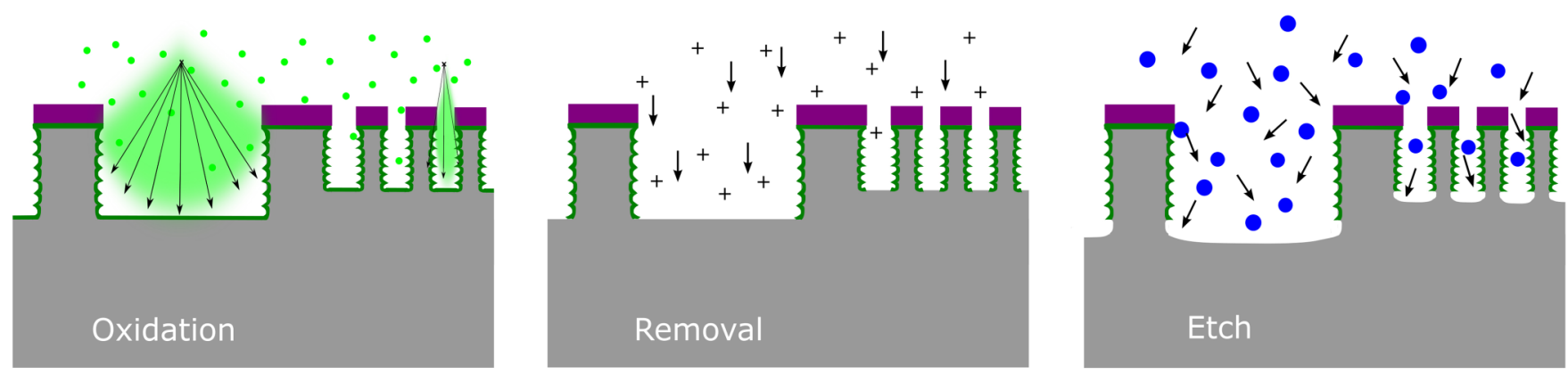

b. CORE process



FIG. 2. Schematic illustration of BSi formation in the Bosch process (a) and BSi-free CORE process (b). 
species is smaller in high aspect ratio features than in the open field area due to neutral shadowing. ${ }^{38}$ This leads to "deposition lag" as the incoming species hit and stick, which means that the open field area will accumulate more deposits than the high aspect ratio features. The next step, the R-step, is controlled by the directional ion flux and, therefore, the removal rate will slightly depend on the ion angular distribution (IAD). ${ }^{38-40}$ In this step, the pressure is so low that it will limit collisions inside the dark space, thus preventing broadening of the IAD. In addition, a low pressure will raise the electron temperature and consequently raises the plasma potential. ${ }^{41}$ This, in turn, will increase the ratio of the directed ion energy gained in the sheath to the random ion energy in the plasma, resulting in a sharper IAD. ${ }^{42}$ Therefore, the R-step is considered to be less dependent of the aspect ratio. As a result, exactly after the FC layer at the bottom of high aspect ratio features is totally removed, there are still some residues remaining in the open field area [Fig. 2(a2)]. This FC residue will initiate BSi when the time to undercut these residues in the next E-step is too short [Fig. 2(a3)]. The E-step is, like the deposition step, highly aspect ratio dependent due to Knudsen transport limitations causing depletion. It means that in the free molecular regime, random reflections of the passivated sidewalls will increasingly restrict the number of etching species to reach the bottom. This is the mechanism behind the well-known RIE lag.

In contrast, the plasma oxidation process of the CORE sequence is self-limiting [Fig. 2(b1)]. This characteristic allows the oxide layer to grow with almost the same thickness everywhere either in the high aspect ratio areas or in the large open field areas. The oxide layer in the open field areas is always a little bit thicker than inside the high aspect ratio features. However, the difference in absolute thickness will be less when the oxidation time increases due to the logarithmic film growth. Therefore, the deposition lag associated with the Bosch process is virtually not present in the CORE process. As a result, in the upcoming R-step [Fig. 2(b2)] being also almost independent of the aspect ratio, all passivation at the horizontal surfaces will be cleared almost at the same time independent of the aspect ratio. Figure 3 is an illustrative demonstration of Fig. 2 to highlight the difference between the Bosch and CORE processes on the formation of $\mathrm{BSi}$. Two samples with increasing linewidths were etched to almost the same depth $(4 \mu \mathrm{m})$ using the Bosch and CORE processes, respectively. It can be seen that with the Bosch process, there is BSi formation in the large open field area due to surface residues while the bottom surface is clean in the high aspect ratio features. This aspect ratio dependent phenomenon usually does not show up as strong in case of the CORE process in which the etch sample has a clean and smooth bottom surface everywhere [Fig. 3(b)].

Although the CORE sequence has been developed for BSi-free applications, it is also possible to modify the recipe to create BSi for other applications such as solar cells. This request can be addressed due to the flexibility of the CORE parameters. It is noticed in Fig. 1 that there are four distinct steps in the CORE sequence and parameters in each step can be changed independently to investigate the formation of BSi. The advantage of using the CORE sequence is that we can combine different parameters to control when surface roughening occurs and then manipulate the parameters to either enhance or suppress the BSi appearance. In this study, the BSi formation will be investigated by first taking the most straightforward technique by limiting the R-time to ensure that not all the passivation at the horizontal surfaces is removed after the ion bombardment. After that, other options will follow.

\section{B. R-step}

To create the requested directionality with a smooth surface and reasonable mask selectivity, the removal time is adjusted typically to be just enough to clear away the oxide layer on the horizontal surfaces. But, if the removal time is too short, residues will still remain at random spots on the bottom surface and act as micromasks. In the subsequent E-step, and assuming the etch time to be too short to fully undercut the self-inflicted micromasks, local roughness in the silicon will initiate and strengthen further in the upcoming cycles, until sharp needlelike structures (BSi) appear. This formation mechanism is explained in more detail in Fig. 4.

In the first row [Figs. 4(a)-4(d)], where the E-step time is taken quite long $(73 \mathrm{~s})$, it is observed that the R-time of $15 \mathrm{~s}$ is sufficient to prevent BSi [Figs. 4(a) and 4(b)]. In case of shorter R-times [Figs. 4(c) and 4(d)], the removal and undercut is insufficient and larger oxide residues will remain. It is also noticed that when BSi appears [Figs. 4(c) and 4(d)], the resist mask is considerably more undercut than when BSi is absent [Figs. 4(a) and 4(b)]. The reason is that when BSi is formed, the etch rate will drastically slow down and less fluorine will be consumed. Consequently, the fluorine pressure is getting higher and thus will erode more the sidewall of the etching feature. The same happens when the E-time is shortened to $35 \mathrm{~s}$, as shown in the second row [Figs. 4(e)-4(h)]. However, in this case, the R-time of $15 \mathrm{~s}$ [Fig. 4(f)] is not enough to prevent $\mathrm{BSi}$ as the undercut is shorter and smaller oxide residues

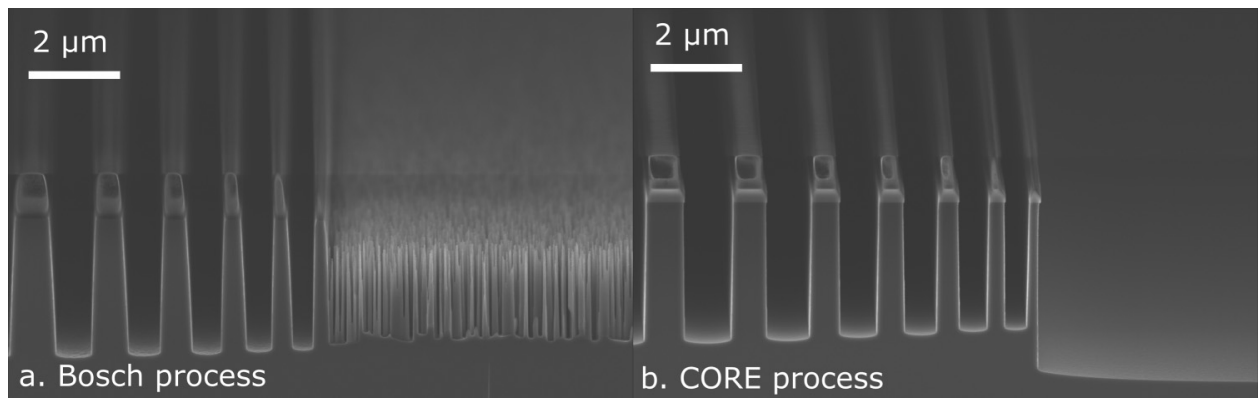

FIG. 3. Samples etched using (a) the Bosch process result in a clean bottom in high aspect ratio features and $\mathrm{BSi}$ in the large open field areas, while (b) the CORE process results in a clean and smooth bottom everywhere. 




FIG. 4. Effect of decreasing R-time on the formation of BSi. C-time is $4 \mathrm{~s}$ and O-time is $3 \mathrm{~s}$.

are able to remain at the surface area. It is noted in this case, just like the Bosch process [Fig. 3(a)], that the open field structures are more likely to form BSi. This is because in this experiment, we only use $3 \mathrm{~s}$ for the O-step, which is not yet operational in the selflimiting regime as shown in our previous study. ${ }^{33}$ Therefore, the open field areas will have a little more passivation and are more likely to develop grass when the R-time is restricted. Again, the formation of BSi in the open field will raise the fluorine pressure and, therefore, we observe a faster etch rate in the high aspect ratio features as these are virtually free of oxide residues. Finally, in the last row [Figs. 4(i)-4(1)], where the E-time is the shortest (15 s), BSi is present even for the longest R-time of $20 \mathrm{~s}$. Remarkably, in this case [Fig. 4(i)], BSi only appears in the higher aspect ratio features while the open field is free of BSi. This time the reason is believed to be caused by Knudsen restrictions of the fluorine species inside the high aspect ratio features that limit the etch rate and undercutting locally, thus preventing very small oxide residues from undercutting. Therefore, in order to get the best BSi-free etch performance with a minimum undercut, it is important to make sure that the passivation is removed from all the horizontal surfaces.

From the previous experiment and discussion, we can conclude that the R-time of $20 \mathrm{~s}$ is not enough to clear all the oxide residues since Fig. 4(i) proves that some very small oxide residues have survived. Therefore, the R-time is increased further as shown in Fig. 5. When the E-time stays at $15 \mathrm{~s}$, like Fig. 4(i), but the
R-time is increased to $25 \mathrm{~s}$ [Fig. 5(a)], the grass indeed disappears. However, decreasing the E-time further to $5 \mathrm{~s}$ and keeping the R-time at $25 \mathrm{~s}$ [Fig. 5(b)] is again developing grass as the tiniest oxide residues survive the ultrashort undercut. Finally, increasing the R-time to $30 \mathrm{~s}$ at $5 \mathrm{~s}$ E-time [Fig. 5(c)] is also removing these tiny leftovers and all the surfaces become smooth.

\section{E-step}

The E-step seems to have no direct contribution to the formation of surface residues as it basically only etches silicon isotropically. However, when oxygen is added in the E-step (as usual in mixed mode RIE) and causing an intimate contact between the etching and passivating species, $\mathrm{BSi}$ is formed. The main reason is believed to be due to a competition between oxygen and fluorine radicals to react with silicon surface atoms. Some areas will be oxidized and stop etching, while other areas are fluorinated and continue to etch. This will cause initial surface roughening. In subsequent steps, this roughness will further enhance to BSi because of the directionality provided by the R-step. Another possibility to form BSi by mixing $\mathrm{SF}_{6}$ with $\mathrm{O}_{2}$ during silicon etching is that the $\mathrm{SiO}_{2}$ plasma dust is created. ${ }^{27}$ These dust particles might settle on the silicon surface and form micromasks. However, the presence of plasma dust has not been verified and, therefore, we will ignore this mechanism in this section. Anyways, $\mathrm{BSi}$ is clearly enhanced by mixing $\mathrm{SF}_{6}$ with $\mathrm{O}_{2}$. 


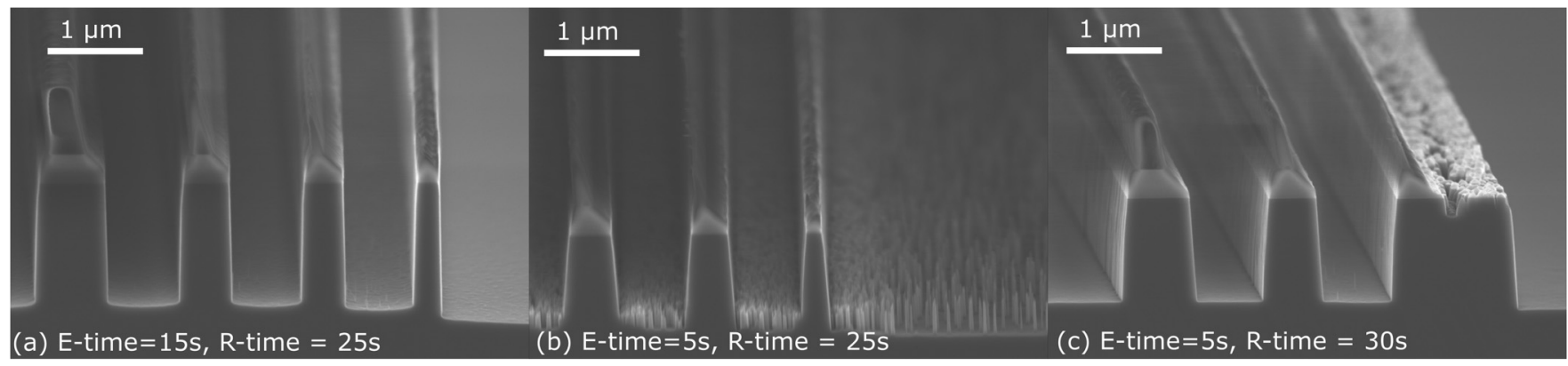

FIG. 5. Relation between R-time and E-time on the formation of BSi. C-time is $4 \mathrm{~s}$ and O-time is $3 \mathrm{~s}$.

Figure 6 shows the result after adding 5,10, and 15 SCCM of $\mathrm{O}_{2}$ into the E-step. In the first row, when E-time is at $73 \mathrm{~s}$, there is no $\mathrm{BSi}$ appearing regardless of the amount of $\mathrm{O}_{2}$ added into the E-step. Seemingly, the long etch time is sufficient to undercut all the micromasks and, therefore, prevent the evolution of $\mathrm{BSi}$. Nevertheless, with the presence of $\mathrm{O}_{2}$ content, the bottom is getting rougher as the oxygen radicals compete with the fluorine radicals to oxidize the silicon randomly. Furthermore, it is noted that when the amount of $\mathrm{O}_{2}$ in the E-step increases, these species will inhibit the etching process increasingly and make it more difficult for the fluoride radicals to penetrate the oxide layer and to react with the silicon. Therefore, the etch rate lowers and the etch profiles become more positive [Figs. 6(a)-6(d)]. Since the etch rate gets lower, the fluorine pressure will rise, and as a result, the lateral etching (undercut) is more pronounced. The same principle applies for $35 \mathrm{~s}$ and $15 \mathrm{~s}$ E-time [Figs. 6(e)-6(1)], but in these cases, BSi appears as the micromasks are big enough to survive the lower undercut. The shorter the E-time, the lower the undercut and the easier it is to get $\mathrm{BSi}$. It is also observed that BSi is more pronounced in the open field areas. The reason is because the

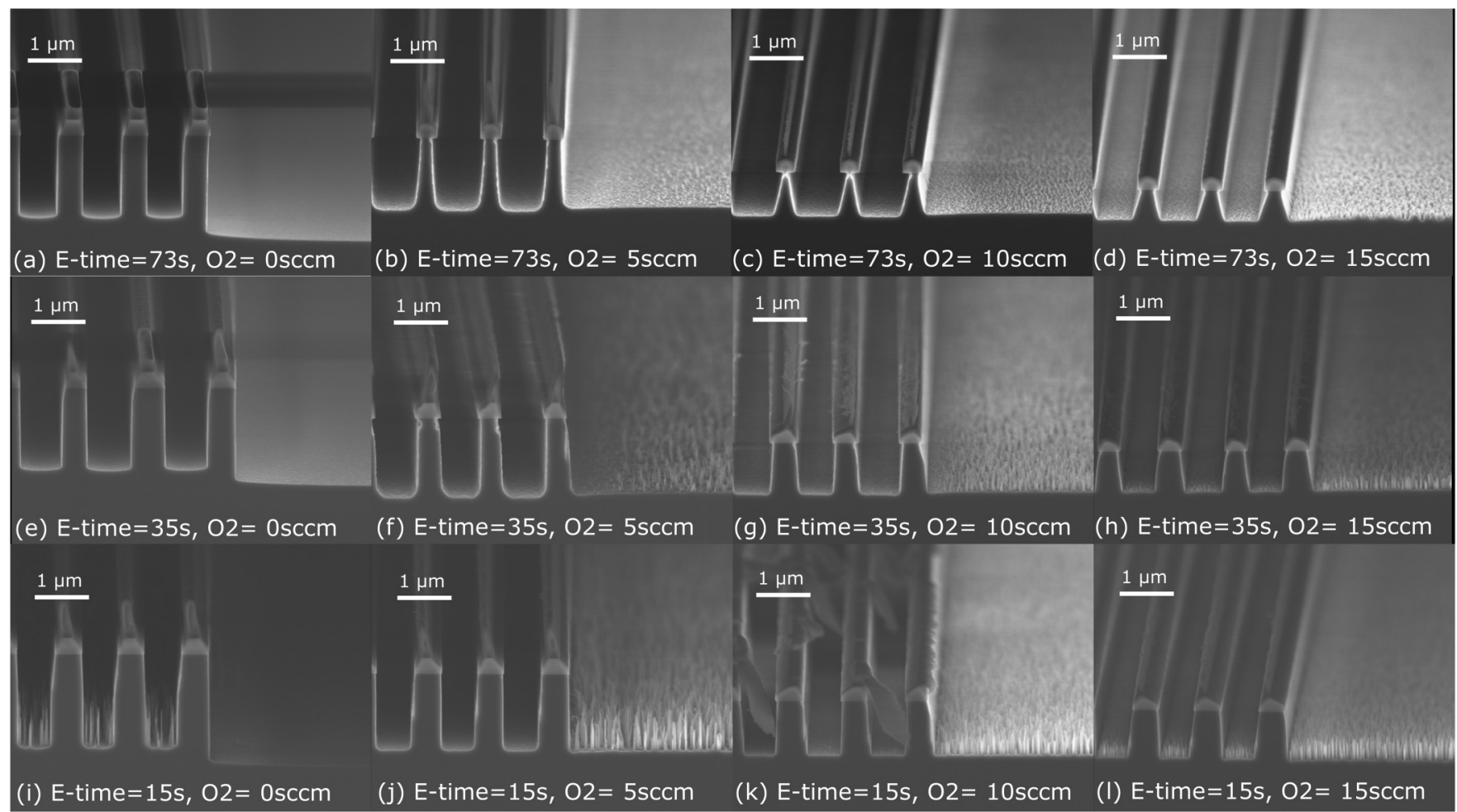

FIG. 6. Effect of adding $\mathrm{O}_{2}$ into the E-step. C-time is $4 \mathrm{~s}$, $\mathrm{O}$-time is $3 \mathrm{~s}$, and R-time is $20 \mathrm{~s}$. 
micromasks responsible for $\mathrm{BSi}$ are probably caused by the local oxidation from the arriving oxygen radicals and thus have a tendency to form more in the open field areas than in the high aspect ratio structures. This is just like the deposition lag mechanism that has been found to be responsible for the BSi formation in the Bosch process [Fig. 2(a)]. Therefore, even though the method of adding $\mathrm{O}_{2}$ into the E-step can create $\mathrm{BSi}$, it is not what we aim for. BSi formation is not uniform as more BSi appears in the large open field area than in the high aspect ratio structures.

\section{O-step}

Another option to manipulate BSi formation is changing the O-step. During the O-step, the F-terminated silicon surface created during the earlier E-step will be replaced by the O-terminated silicon surface. This plasma oxidation process is essential to enable anisotropic etching. More to the point, oxide growth slows down logarithmically once it is formed and becomes self-limiting when roughly $3 \mathrm{~nm} \mathrm{SiO}$ xassivation has grown. ${ }^{33}$ The latter means that at longer oxidation times, all surfaces tend to have exactly the same protective layer thickness independent of the aspect ratio. For the shorter O-step times, though, the limitation is not yet completed and the open areas will have more passivation than the high aspect ratio features and can result in BSi when the R-step is insufficient [as previously observed in Fig. 4(f)]. Therefore, in order to have $\mathrm{BSi}$ formation on all locations, the O-step time should be enough to form an almost identical layer on any topography. The higher the differences in aspect ratio the longer O-time is needed to ensure that the self-limiting regime is reached.

\section{E. C-step}

The last option to manipulate BSi formation we studied is the change in C-step. As already introduced in Sec. II, the C-step is included to prevent the $\mathrm{SF}_{6}$ plasma from the preceding E-step to come into contact with the $\mathrm{O}_{2}$ plasma in the next O-step. Although the $\mathrm{SF}_{6}$ flow has stopped in this step, the $\mathrm{SF}_{6}$ gas still has a certain residence time in the reactor. For example, for a plasma volume $\mathrm{V}=3 \mathrm{l}$ with a flow $\mathrm{Q}=15 \mathrm{SCCM}(\sim 25 \mathrm{Pal} / \mathrm{s})$ and a pressure $\mathrm{p}=50 \mathrm{mT}(\sim 7 \mathrm{~Pa})$, the residence time is about $\mathrm{t}_{\text {res }}=\mathrm{V} \times \mathrm{p} / \mathrm{Q}=1 \mathrm{~s}^{18}$ If there is no such $\mathrm{C}$-step, the $\mathrm{O}$-step will contain $\mathrm{SF}_{6}$ that allows for etching. Consequently, the passivation layer becomes weaker and, therefore, the etch profile is strongly eroded and undercut. This undercutting will prevent BSi formation even though micromasking might be present. Decreasing the etch time to 35 and $15 \mathrm{~s}$ [Figs. 7(c) and $7(\mathrm{~d})$ ] will help reduce the erosion of the structures sidewall as expected. The profile looks great without undercut and does not form $\mathrm{BSi}$. The observation that no BSi is formed when the E-time is very short and the C-time is omitted [Fig. 7(d)] makes us to believe that the CORE cycle does not generate plasma dust that could have initiated BSi. In conclusion, this method has failed to create aspect ratio independent $\mathrm{BSi}$ and is consequently not suited for BSi on demand.

\section{F. Black silicon on demand}

We have demonstrated in Secs. III B-III D the freedom to tune parameters of the CORE sequence to uniformly create $\mathrm{BSi}$-free or BSi-full surfaces. For the BSi-free recipe, the etch process should follow the fine-tuned parameters listed in Fig. 1 (or an adapted version arrived from the results shown in Figs. 4 and 5). This ensures that the etch profiles always have clear and smooth bottom surfaces, both in the high aspect ratio structures and open field area. With respect to the BSi-full recipe, it is shown that there are several ways to modify the CORE parameters for the generation of BSi. However, the most easy and straightforward way is to decrease the removal time in the R-step. This approach facilitates the existence of micromasks everywhere independent of the aspect ratio, which then further evolves into BSi.

Figure 8(a) shows silicon pillars having $1 \mu \mathrm{m}$ diameter that are etched with a BSi-free recipe. The structures are smooth and directional. By combining BSi-free and BSi-full recipes, sequentially interesting features can be created. Figure 8(b) is an example of silicon pillars etched first with a BSi-free recipe and then continued with a BSi-full recipe. This combination results in high aspect ratio pillars with BSi coverage at the bottom. By removing the mask on top of the pillars, the BSi can be formed on top of the these pillars as well [Figs. 8(c)-8(h)]. This proves that the CORE sequence has the ability to form BSi everywhere, either at the bottom of high aspect ratio features or on top of it (the latter resembles an open field feature). It can be observed that, regardless of the dimension of the design, the bottom of the etched features always have the same property, which is either clean and smooth [Fig. 8(a)] or with BSi everywhere [Figs. 8(c)-8(h)]. This advantage outperforms the CORE process over other RIE techniques like the Deposit, Remove, Etch, Multistep or Bosch process, with the latter having the

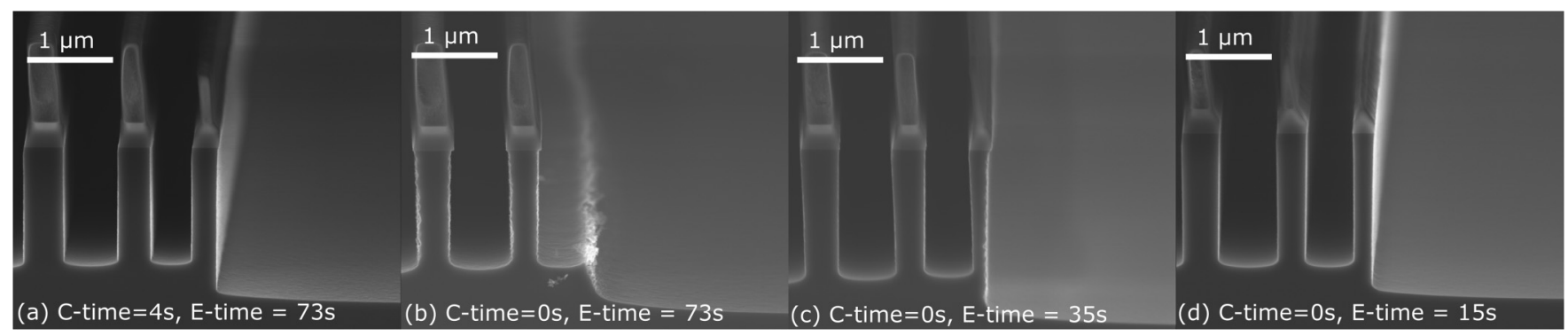

FIG. 7. Effect of decreasing C-time on the formation of BSi. O-time is $3 \mathrm{~s}$ and R-time is $20 \mathrm{~s}$. 




FIG. 8. Silicon pillars etched with (a) BSi-free recipe, (b) BSi-free recipe followed by a BSi-full recipe. (c)-(h) The same double etch process, but the mask is stripped in between. 
tendency to grow more grass in the open field areas than inside the high aspect ratio features.

\section{CONCLUSIONS}

This study is built on the CORE sequence in which the usual FC inhibitor of the Bosch process is replaced by oxygen. Due to the self-limiting property of the oxidation step, the formation and controllability of BSi in the CORE sequence is different from how BSi presents itself in FC-based sequences. The effects of different process parameters on the creation of masks and the formation of BSi have been carefully investigated. It is concluded that the CORE sequence is able to create $\mathrm{BSi}$ or micrograss at will and there is a strong relationship between the R-time and E-time on its formation. The usual function of the R-time is to remove any surface passivation while the E-time should undercut possible leftovers. This will result in smooth surfaces. However, if we restrict the R-time to leave oxide residue and use an E-time short enough to prevent undercut of this residue, BSi will develop. The advantage of this method and using the CORE sequence is that BSi is created with the same properties everywhere: it covers horizontal surfaces either in and on top of high aspect ratio structures or in large open field areas. The latter distinguishes the BSi formation clearly from other directional processes such as the Bosch process. The proposed CORE process thus provides the authors a versatile tool for creating BSi anywhere anytime or-as we call it_-BSi on Demand."

\section{ACKNOWLEDGMENTS}

The authors would like to thank the DTU Nanolab staff for instrument support. Particularly, they thank Lean Gottlieb Pedersen and Thomas Aarøe Anhøj for the support with maskless lithography and Roy Cork and Martin Nørvang Kristensen for technical support with plasma etching processes.

\section{REFERENCES}

${ }^{1}$ X. Liu, P. R. Coxon, M. Peters, B. Hoex, J. M. Cole, and D. J. Fray, Energy Environ. Sci. 7, 3223 (2014).

2J. S. Yoo, I. O. Parm, U. Gangopadhyay, K. Kim, S. K. Dhungel, D. Mangalaraj, and J. Yi, Sol. Energy Mater. Sol. Cells 90, 3085 (2006).

${ }^{3}$ J. Yoo, G. Yu, and J. Yi, Sol. Energy Mater. Sol. Cells 95, 2 (2011).

${ }^{4}$ P. Repo, J. Benick, V. Vähänissi, J. Schön, G. von Gastrow, B. Steinhauser, M. C. Schubert, M. Hermle, and H. Savin, Energy Procedia 38, 866 (2013).

5J. Rong, C. Masarapu, J. Ni, Z. Zhang, and B. Wei, ACS Nano 4, 4683 (2010).

${ }^{6}$ M. Steglich, M. Zilk, A. Bingel, C. Patzig, T. Käsebier, F. Schrempel, E. B. Kley, and A. Tünnermann, J. Appl. Phys. 114, 183102 (2013).

${ }^{7}$ M. Steglich, M. Zilk, F. Schrempel, A. Tünnermann, and E. B. Kley, Appl. Phys. Lett. 102, 111110 (2013).

${ }^{8}$ E. P. Ivanova et al., Nat. Commun. 4, 2838 (2013).

${ }^{9}$ D. Qi, N. Lu, H. Xu, B. Yang, C. Huang, M. Xu, L. Gao, Z. Wang, and L. Chi, Langmuir 25, 7769 (2009).

${ }^{10} \mathrm{M}$. Barberoglou, V. Zorba, A. Pagozidis, C. Fotakis, and E. Stratakis, Langmuir 26, 13007 (2010).

${ }^{11}$ E. Sarajlić, M. J. de Boer, H. V. Jansen, N. Arnal, M. Puech, G. Krijnen, and M. Elwenspoek, J. Micromech. Microeng. 14, S70 (2004).

${ }^{12}$ Y. Zhao, E. Berenschot, H. Jansen, N. Tas, J. Huskens, and M. Elwenspoek, Microelectron. Eng. 86, 832 (2009).
${ }^{13}$ J. Elders, H. V. Jansen, M. Elwenspoek, and W. Ehrfeld, Proceedings IEEE Micro Electro Mechanical Systems, 1995, Amsterdam, Netherlands, 29 January-2 February 1995 (IEEE, New York, 1995), p. 238.

${ }^{14}$ Y. Zhao, E. Berenschot, M. De Boer, H. Jansen, N. Tas, J. Huskens, and M. Elwenspoek, J. Micromech. Microeng. 18, 064013 (2008).

${ }^{15}$ S. Vanapalli, H. J. ter Brake, H. V. Jansen, J. F. Burger, H. J. Holland, T. T. Veenstra, and M. C. Elwenspoek, J. Micromech. Microeng. 17, 1381 (2007).

${ }^{16} \mathrm{M}$. de Boer, H. Jansen, and M. Elwenspoek, Proceedings of the International Solid-State Sensors and Actuators Conference-TRANSDUCERS'95, Stockholm, Sweden, 25-29 June 1995 (IEEE, New York, 1995), Vol. 1, p. 565.

${ }^{17}$ M. C. Louwerse, H. V. Jansen, M. N. W. Groenendijk, and M. C. Elwenspoek, J. Micromech. Microeng. 19, 045008 (2009).

${ }^{18}$ H. V. Jansen, M. J. de Boer, S. Unnikrishnan, M. C. Louwerse, and M. C. Elwenspoek, J. Micromech. Microeng. 19, 033001 (2009).

${ }^{19}$ C. Rusu et al., J. Microelectromech. Syst. 10, 238 (2001).

${ }^{20}$ H. V. Jansen, M. J. de Boer, M. A. Boer, A. M. Otter, and M. C. Elwenspoek, IEEE Workshop on Micro Electro Mechanical Systems, MEMS 1995, An Investigation of Micro Structures, Sensors, Actuators, Machines and Systems, Amsterdam, Netherlands, 29 January-2 February 1995 (IEEE, New York, 1995), p. 88 .

${ }^{\mathbf{2 1}}$ H. Jansen, H. Gardeniers, M. de Boer, M. Elwenspoek, and J. Fluitman, J. Micromech. Microeng. 6, 14 (1996).

${ }^{22}$ F. Zhu, X. Zhang, and H. Zhang, Sci. China Technol. Sci. 58, 381 (2015).

${ }^{23}$ C. M. Silvestre, V. Nguyen, H. Jansen, and O. Hansen, Microelectron. Eng. 223, 111228 (2020)

${ }^{\mathbf{2 4}}$ H. Jansen, M. de Boer, R. Legtenberg, and M. Elwenspoek, J. Micromech. Microeng. 5, 115 (1995).

${ }^{\mathbf{2 5}}$ F. Laermer, F. and A. Schilp, U.S. Patent No. 6,531,068 (11 March 2003).

${ }^{26}$ M. Kroll, T. Käsebier, M. Otto, R. Salzer, R. Wehrspohn, E. B. Kley, A. Tünnermann, and T. Pertsch, Proc. SPIE 7725, 772505 (2010).

${ }^{27}$ H. V. Jansen, M. J. de Boer, K. Ma, M. Girones, S. Unnikrishnan, M. C. Louwerse, and M. C. Elwenspoek, J. Micromech. Microeng. 20, 075027 (2010).

${ }^{28}$ G. Kumaravelu, M. M. Alkaisi, A. Bittar, D. MacDonald, and J. Zhao, Curr. Appl. Phys. 4, 108 (2004).

${ }^{29}$ R. Dussart, X. Mellhaoui, T. Tillocher, P. Lefaucheux, M. Volatier, C. Socquet-Clerc, P. Brault, and P. Ranson, J. Phys. D Appl. Phys. 38, 3395 (2005).

${ }^{30} \mathrm{P}$. Dixit and J. Miao, J. Electrochem. Soc. 153, G771 (2006).

${ }^{31}$ J. T. Drotar, Y. P. Zhao, T. M. Lu, and G. C. Wang, Phys. Rev. B 61, 3012 (2000).

${ }^{32}$ M. Gaudig, M. Maiberg, M. Plapp, and R. B. Wehrspohn, J. Appl. Phys. 124, 233302 (2018).

${ }^{33}$ V. T. H. Nguyen et al., ECS J. Solid State Sci. Technol. 9, 024002 (2020).

${ }^{34}$ B. Chang, P. Leussink, F. Jensen, J. Hübner, and H. Jansen, Microelectron. Eng. 191, 77 (2018).

${ }^{35}$ B. Chang, F. Jensen, J. Hübner, and H. Jansen, J. Micromech. Microeng. 28, 105012 (2018).

${ }^{36}$ B. Chang, Y. Tang, M. Liang, H. Jansen, F. Jensen, B. Wang, K. Mølhave, J. Hübner, and H. Sun, ChemNanoMat 5, 92 (2019).

${ }^{37}$ B. Chang, C. Zhou, A. T. Tarekegne, Y. Yang, D. Zhao, F. Jensen, J. Hübner, and H. Jansen, Adv. Opt. Mater. 7, 1801176 (2019).

${ }^{38}$ A. D. Bailey III and R. A. Gottscho, Jpn. J. Appl. Phys. 34, 2083 (1995).

${ }^{39} \mathrm{H}$. Jansen, M. de Boer, and M. Elwenspoek, Proceedings of Ninth International Workshop on Micro Electromechanical Systems, San Diego, CA, 11-15 February 1996 (1996), p. 250.

${ }^{40}$ H. Jansen, M. de Boer, R. Wiegerink, N. Tas, E. Smulders, C. Neagu, and M. Elwenspoek, Microelectron. Eng. 35, 45 (1997).

${ }^{41}$ M. A. Lieberman and A. J. Lichtenberg, Principles of Plasma Discharges and Materials Processing (Wiley, New York, 2005).

${ }^{42}$ E. S. Aydil, B. O. M. Quiniou, J. T. C. Lee, J. A. Gregus, and R. A. and Gottscho, Mater. Sci. Semicond. Process. 1, 75 (1998). 